\title{
In Situ and Quantitative Tensile Study of Deformation Twinning in Submicron- Sized Mg Crystals Inside a Transmission Electron Microscope
}

Boyu Liu, ${ }^{*}$ Zhiwei Shan, * Xiyan Zhang, ${ }^{* *}$ Jun Sun,* and Evan Ma,***

* Center for Advancing Materials Performance from the Nanoscale (CAMP-Nano) \& Hysitron Applied Research Center in China (HARCC), State Key Laboratory for Mechanical Behavior of Materials, Xi'an Jiaotong University, Xi’an 710049, China, zwshan@mail.xjtu.edu.cn ** School of Materials Science and Engineering, Chongqing University, Chongqing 400044, China *** Department of Materials Science and Engineering, The Johns Hopkins University, Baltimore, Maryland 21218, ema@jhu.edu

Quantitative tests inside the transmission electron microscope (TEM) provide in situ observation and stress-strain response simultaneously during sample deformation. Despite plentiful in situ TEM compression tests [1-4], rare tensile test which is more standard and informative, has been published in the literature so far, especially for HCP materials [5-7,10]. In this report, in situ tension and compression studies have been conducted quantitatively on submicron $\mathrm{Mg}$ single crystal samples, with a Hysitron PI95 TEM holder, for tensile loading in the c-direction and subsequently for compressive loading on the deformed sample. This in situ experiment documents the first direct observation of $\{10-12\}$ twinning that instantly reorients the crystal by nearly $90^{\circ}$, as shown in Fig. 1, and de-twinning that gradually rotates the crystal to its original orientation. In c-axis tension, $\{10-12\}$ twinning remains predominant deformation mechanism when sample size is reduced to D 200 nm, with the twinning stress far exceed that known for bulk Mg [8,9]. Different from compressive loading perpendicular to the c-axis, the tensile loading along the c-axis activates the $\{10-12\}$ twinning at lower stresses [10] and accomplishes the twinning across the entire sample in one major strain burst (high twinning speed), as shown in Fig. 2. The compressive loading on the twinned sample can lead to a de-twinning and the twin boundary propagates from top to bottom gradually, accompanying a clearer work hardening than that in the c-axis tension [11].

References

[1] S. H. Oh, M. Legros, D. Kiener, and G. Dehm, Nat. Mater. 8 (2009) 95.

[2] Z. W. Shan, G. Adesso, A. Cabot, M. P. Sherburne, S. A. S. Asif, O. L. Warren, D. C. Chrzan, A. M. Minor, and A. P. Alivisatos, Nat. Mater. 7 (2008) 947.

[3] Z. W. Shan, R. K. Mishra, S. A. S. Asif, O. L. Warren, and A. M. Minor, Nat. Mater. 7 (2008), 115.

[4] L. D. Marks, O. L. Warren, A. M. Minor, and A. P. Merkle, MRS Bull. 33 (2008) 1168.

[5] Qian Yu, Zhi-Wei Shan, Ju Li, Xiaoxu Huang, Lin Xiao, Jun Sun, and Evan Ma, Nature 463 (2010) 335.

[6] C. M. Byer, B. Li, B. Y. Cao, and K. T. Ramesh, Scr. Mater. 62 (2010) 536.

[7] E. Lilleodden, Scr. Mater. 62 (2010) 532.

[8] Wonsiewi.Bc and W. A. Backofen, Transactions of the Metallurgical Society of Aime. 239 (1967) 1422.

[9] M. R. Barnett, Mater. Sci. Eng. A-Struct. Mater. Prop. Microstruct. Process. 464 (2007) 1.

[10] J. Ye, R. K. Mishra, A. K. Sachdev, and A. M. Minor, Scr. Mater. 64 (2011) 292.

[11] This work was supported by the grants from NSFC (50720145101, 50831004 and 50925104) and 973 Program of China (2010CB631003). We also appreciate the support from the 111 
Project of China (B06025). The work of E. M. was carried out under an adjunct professorship at XJTU and supported in part by USNSF-DMR-0904188.



FIG. 1. (a) Bright-field TEM images (overlapped still frames, taken from the recorded movie) showing the sample before and after twinning, (b) Diffraction patterns showing the twin relationship.

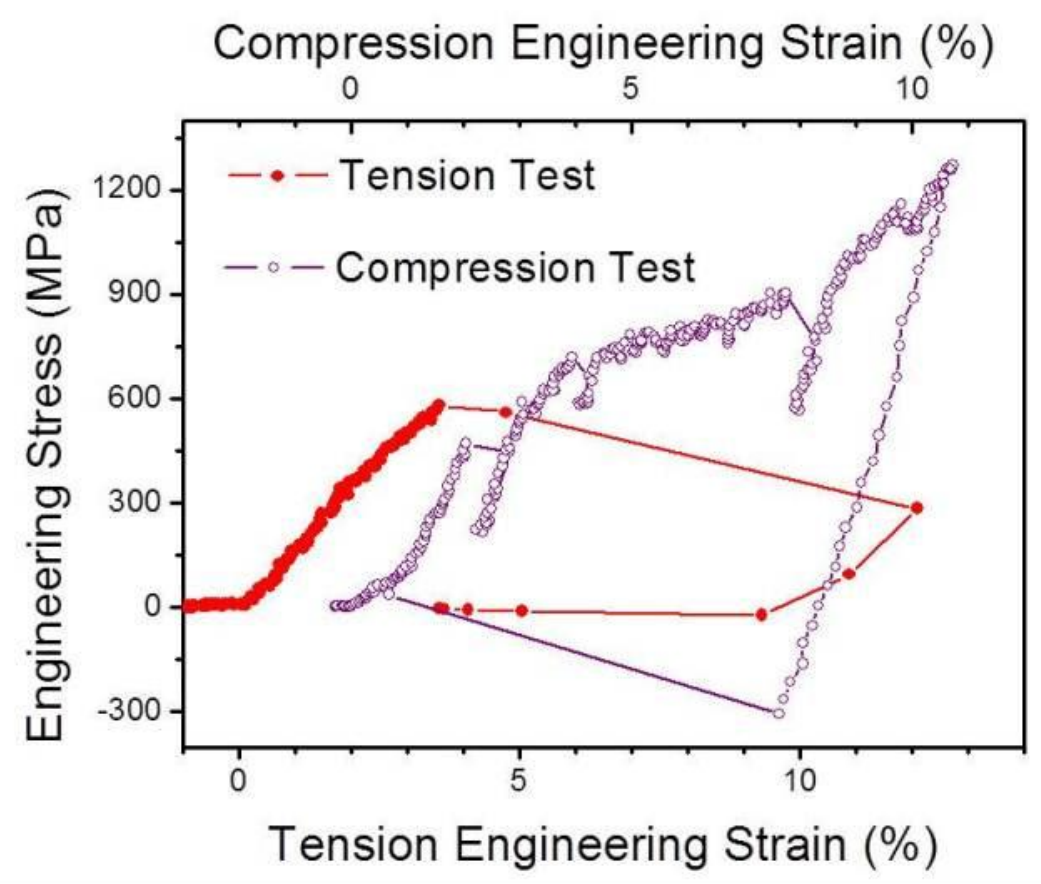

FIG. 2. Engineering stress-strain curves comparing the $\{10-12\}$ twinning behavior activated by tension along the c-axis versus the de-twinning activated by compression perpendicular to the c-axis. 